\title{
Bidirectional DC-AC Converter-Based Communication Solution for Microgrid
}

Research Article

\author{
Yixuan Zhang ${ }^{1}$, Kai $\mathrm{Ni}^{2}$, Yangang Wang ${ }^{3}$, Yihua $\mathrm{Hu}^{1, *}$ \\ 'Department of Electronics, University of York, York, United Kingdom \\ ${ }^{2}$ School of Electrical and Electronic Engineering, Huazhong University of Science and Technology, Wuhan, China \\ ${ }^{3}$ Dynex Semiconductor Ltd., Doddington Road, Lincoln, United Kingdom
}

Received: October 15, 2020; Accepted: November 27, 2020

\begin{abstract}
The communication system of a microgrid can transfer the information of electricity price, power consumption and so on between users and the control centre. This capability is of great significance to improve the efficiency and sustainability of power facilities. In this paper, a bidirectional DC-AC converter topology is proposed to achieve the composite transmission of power and signals in microgrids. Since the transmitted signals are modulated by power switches of converters and integrated into the currents, the cost of signal couplers can be saved and the circuit structure can be simplified. In order to verify the feasibility of the proposed method, a simulation model of the proposed converter is implemented in MATLAB/Simulink. With the power supply frequency of $50 \mathrm{~Hz}$, when the converter operates in the inverter mode and rectifier mode, the data transmission rate can reach $120 \mathrm{bit} / \mathrm{s}$ and 48 bit/s, respectively.
\end{abstract}

Keywords: bidirectional DC-AC converter • frequency shift keying $\bullet$ MATLAB simulation • microgrid $\bullet$ two-way communication

\section{Introduction}

Given the requirements of high reliability and low cost in developing power grid systems, the concept of microgrid is proposed to realise large-scale energy conversion and power transmission through resource optimisation (Tripathi et al., 2020). The microgrid system takes advantage of the two-way flow of power and information to achieve realtime demand-supply regulation in a consistent, secure and efficient manner (Fang et al., 2011; Hau et al., 2013; Mocanu et al., 2016; Sun et al., 2011). Since a large amount of data are generated and transmitted in microgrids, an efficient information transmitting approach is essential for not only dealing with such massive data but also for addressing network security issues such as customers' information leakage and large-scale blackout (Ghorbanian et al., 2019; Lu et al., 2010).

Although the existing communication technologies which are applied in microgrids can satisfy the requirements of data exchange, each method has its limitations. For instance, an optical fibre can provide $100 \mathrm{Mbps}$ to $40 \mathrm{Gbps}$ data rates at the access layer, core level and aggregation level, which makes it popular in the field of public utilities (Fang et al., 2011; Lo and Ansari, 2011). However, if there is no existing infrastructure, especially in rural areas, utilising such a technique will cause slow deployment and high installation costs. Besides, wireless technologies such as cellular networks and Wi-Fi networks are well-developed communication approaches. With the merits of large coverage, fast response and low operational and maintenance costs, wireless communication is applicable for real-time applications in urban areas (Gungor et al., 2011; Wang et al., 2011). However, the associated high interference-sensitivity is a major weakness characterising wireless communication because of its unfixed communication channel (Gungor et al., 2011; Wang et al., 2011). Furthermore, power line communication (PLC) is the technology that employs power lines as the transmission medium for both power and communication signals. Since bulky communication cables are omitted in this approach, it has higher mobility and flexibility for end 
devices. Nevertheless, the attenuation, distortion and noise are critical issues for the PLC-based radio frequency communication, which make it difficult for the data rates of narrowband PLC and broadband PLC to exceed $500 \mathrm{Kbps}$ and $200 \mathrm{Mbps}$, respectively (Gungor et al., 2011; Kabalci, 2016; Lo and Ansari, 2011; Wang et al., 2011).

In the conventional PLC-based microgrid, capacitive coupler, inductive coupler and resistive coupler are commonly employed to couple signals to the power cable (Costa et al., 2017). In capacitive coupling, one or more capacitors are series connected with the power cable to provide a low-impedance path for high-frequency components of the power line signal (Costa et al., 2017). The capacitive coupler is widely employed in low-voltagebased PLC systems because of its low insertion loss (Costa et al., 2015). In an inductive PLC coupler, the current flows through the winding coil and generates an electromagnetic field that induces the signal into the conductor (Lee et al., 2010). The inductive couplings are suitable for medium-voltage-based PLC systems, since they have the capability for electromagnetic isolation between the PLC transceiver and the power cable (Costa et al., 2017). With their simple circuitry and low-cost features, resistive couplers are suitable for low-voltage-based PLC systems (Swana et al., 2015). However, resistive couplers are only practicable for signal extraction from the power cable as a result of the intrinsic nature of conductive properties (Swana et al., 2015). Although these coupling approaches can efficiently transmit data through cables, the increased cost and the complexity of the system structure needs to be taken into account prior to the usage of additional couplers in microgrids.

In order to simplify the method of applying couplers to couple signals to the power line, some signal and energy integration approaches have been explored in related researches. Stefanutti et al. $(2006,2008)$ proposed the idea of using the internal power switch of a converter to achieve data and energy composite modulation. Without additional coupling circuits, data modulation is realised only by controlling the frequency or phase parameters of the power switches. Wang et al. (2016) combines direct sequence spread spectrum and phase shift keying modulation strategies, and uses the boost and buck converter voltage ripple as a carrier to achieve synchronous transmission of data and energy while reducing electromagnetic interference. According to Choi and Jung (2017), the power flow information sharing between the converters is realised by modulating the switching frequency of the converter and loading the power flow information of the microgrid on the voltage ripple of the DC bus. This approach is beneficial since it helps to increase the light load efficiency and zero voltage switching capacity of the dual active bridge converter. Since most of these researches investigate the data and energy composite modulation strategies of DC systems, and the application of this technology to the AC microgrid can not only simplify the system structure, but also save costs, the relevant modulation methods in AC microgrids are still worthy of further study.

This paper proposes a bidirectional DC-AC converter topology for two-way communication in AC microgrids. In this design, power conversion is achieved by a full-bridge converter, and the frequency shift keying (FSK) approach is used to modulate the transmission signals. Since the transmitted data are modulated by the switching frequency of the converter instead of applying additional coupling circuits in the proposed approach, the infrastructure cost and structural complexity of the microgrid can be greatly reduced. Moreover, the coupling noise can be eliminated by using this transmission method.

The rest of this paper contains the following contents. Section 2 presents the topology of the proposed converter. Section 3 describes the mechanisms of power transmission and signal transmission. The simulation results are exhibited in Section 4. Finally, a brief conclusion is reached in Section 5.

\section{Integrated Power and Signal Transmission Model}

Power electronic converters are essential components in a microgrid. As shown in Figure 1, a microgrid can be compatible with wind, photovoltaic and other power generation technologies to provide power flexibly for the end users. These energy generation methods are mostly based on power electronic converters, to ensure that the same specifications of power are transmitted through the power line (Chakraborty et al., 2007). With the proposed approach, power can be transmitted along with the communication signals through the converters. For instance, the residual power information of the power grid can be transmitted to the controller of energy storage system (ESS) in time through the bidirectional DC-AC converter; thus the ESS can store energy in the low-power consumption periods and return the energy to the grid during the summit-power consumption periods.

The proposed bidirectional DC-AC converter topology is presented in Figure 2. There are two series connected full-bridge converters at the DC side, where the upper converter is applied for signal modulation and the lower one is used for power modulation. In addition, two $A C$ voltage sources $V_{4}$ and $V_{5}$ are connected in series with the 


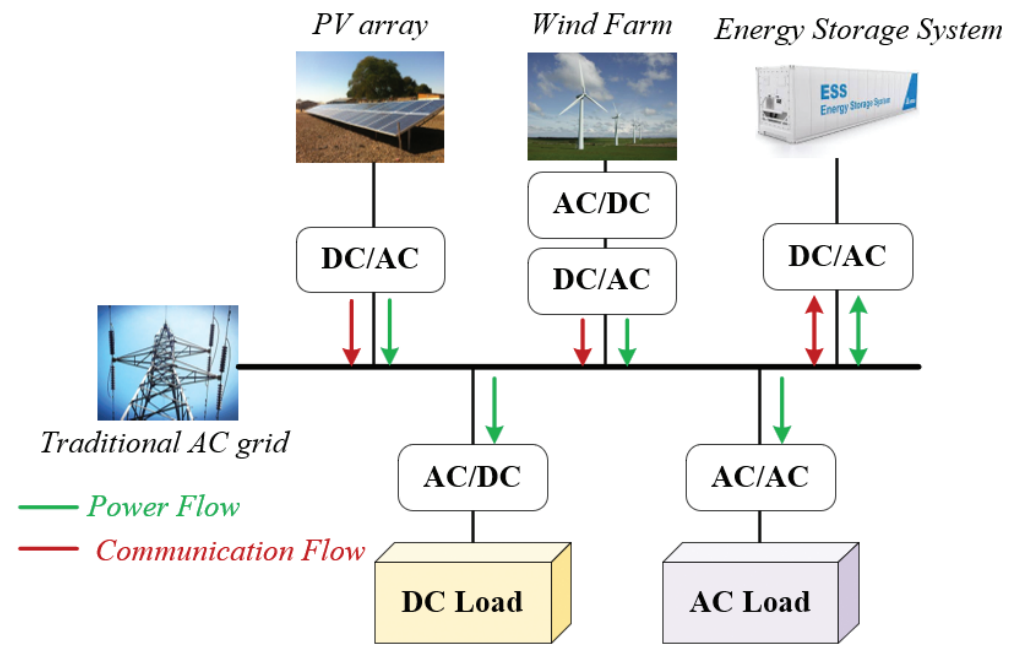

Fig. 1. Conceptual structure of $A C$ microgrid using the proposed power and signal integration strategy.

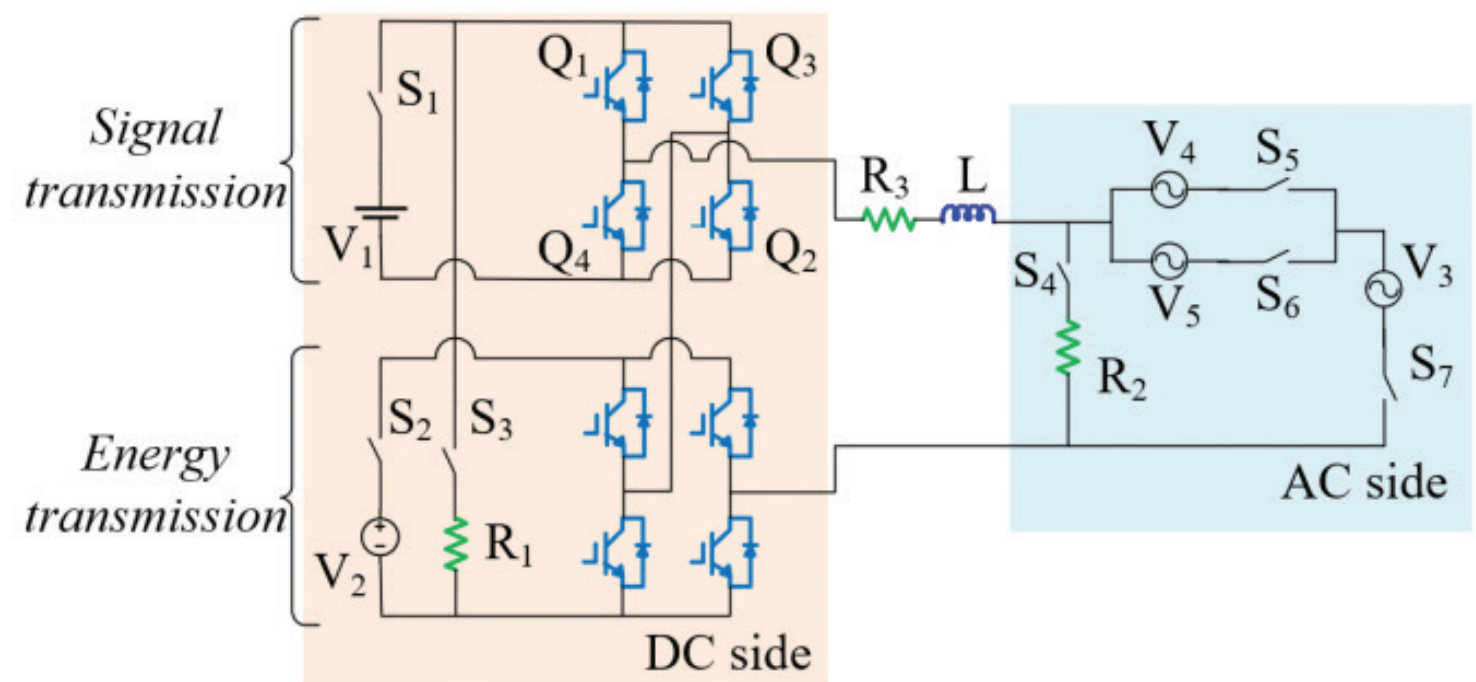

Fig. 2. Schematic of the proposed bidirectional DC-AC converter.

AC power grid $V_{3}$ to generate different frequency carriers. $R_{3}$ and $L$ in series, respectively, represent the inherent impedance and inductance of the transmission line. When the topology operates in the rectifier mode, the switch $\mathrm{S}_{3}$ will turn on to isolate the DC source and the load $\mathrm{R}_{1}$ will be connected to the AC power source. Similarly, when the circuit is operating in the inverter mode, the load $R_{2}$ will be connected to two full-bridge converters to isolate the AC power source.

\section{Operation Mechanisms}

\subsection{Inverter Mode}

The topology of the proposed converter in the inverter mode is shown in Figure 3. In this circuit, the upper fullbridge circuit containing the power supply $\mathrm{V}_{1}$ is used to modulate the transmission signal, and the lower full-bridge circuit involving the power supply $V_{2}$ is employed to modulate $A C$ current. The AC output power is acquired by the 


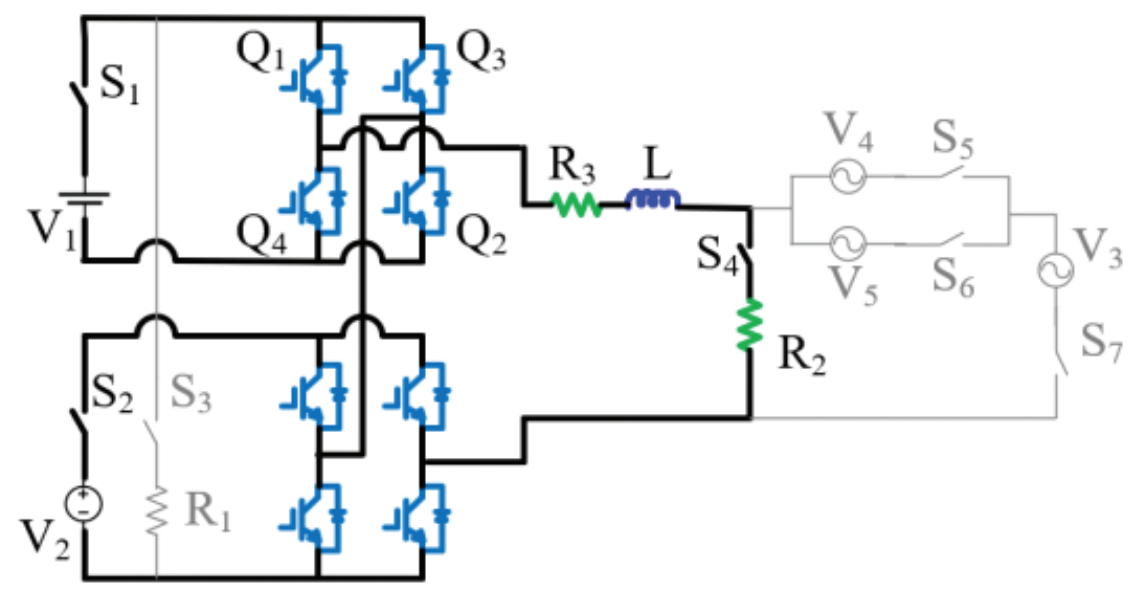

Fig. 3. Schematic of the proposed converter in inverter mode.

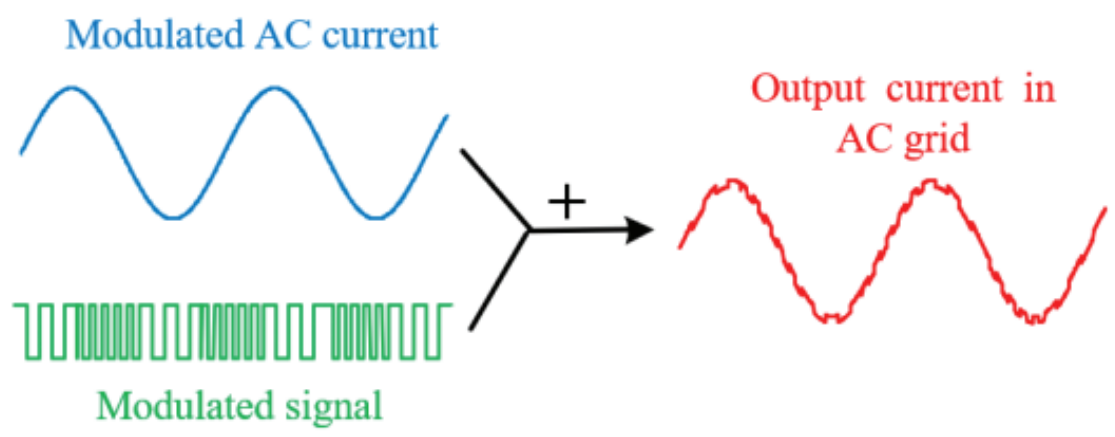

Fig. 4. The relationship between the transmitted signal and the current waveform.

conventional sinusoidal pulse width modulation approach, which uses a reference sine wave to compare it with the triangle carrier and employs the comparison results to control the four IGBT switches. To utilise the FSK method for signal modulation, the high-frequency carriers are generated by fast switching of the four switches of the upper full-bridge converter. For example, if a 4-bit signal '1100' requires to be transmitted, the switches $Q_{1}$ and $Q_{2}$ will turn on and turn off, respectively, at the same time with a specific frequency to modulate ' 1 ' whereas the switches $Q_{3}$ and $Q_{4}$ will operate simultaneously using another frequency to modulate ' 0 '. Furthermore, the working states of the switches $Q_{1}$ and $Q_{2}$ are contrary to those of the switches $Q_{4}$ and $Q_{3}$, respectively, to prevent short-circuiting. Since the two full-bridge converters for signal transmission and energy transmission are connected in series, the modulated signal is superimposed on the modulated current waveform, as exhibited in Figure 4. Since the amplitude of the power source $\mathrm{V}_{1}$ is much smaller than that of the power source $\mathrm{V}_{2}$, the modulated signal is superimposed on the current in the form of small amplitude fluctuation, which will not cause large distortions to the current waveform.

\subsection{Rectifier Mode}

The proposed topology will operate in rectifier mode when the AC power source is connected to the load at the DC side. As shown in Figure 5, all IGBT switches are off, and the current will flow through the anti-parallel diodes of these switches to realise the rectification process. Since the current is transmitted through two series-connected diodes $Q_{1}$ and $Q_{5}$ in Figure 5, the output current waveform obtained in this process is the same as that achieved by the conventional single-diode half-wave rectification method. In this circuit, two AC power supplies $V_{4}$ and $V_{5}$ are utilised to generate different frequency carriers. Specifically, if a 4-bit signal '1100' is transmitted through this topology, the switches $S_{5}$ and $S_{6}$ will turn on to modulate digital ' 1 ' and ' 0 ', respectively. As the power supplies $V_{4}$ 
and $V_{5}$ are connected in series with the grid power source $V_{3}$, the modulated signal is integrated with the $A C$ current and transmitted through the grid. The integration process of transmission signal and power is shown in Figure 6.

\subsection{Demodulation}

After the integrated power and signal are transmitted through the AC grid, an efficient demodulation approach is essential to restore the data at the receiver. The proposed demodulation process is presented in Figure 7 . First, a bandpass filter is used to extract the carrier from the current waveform of the receiver. Because the digital ' 1 ' and ' 0 ' of the transmitted signal are modulated by different frequency carriers, any carrier can be separated from the current by using a suitable bandpass filter. The Fourier series expansion of a random periodic signal $F(x)$ is given by

$$
F(x)=\frac{1}{2} a_{0}+\sum_{n=1}^{\infty}\left(a_{n} \cos n \omega x+b_{n} \sin n \omega x\right)
$$

where $\omega$ is the angular frequency, $T$ is the signal period and the coefficients $a_{0}, a_{n}$ and $b_{n}$ are represented by the following expressions, respectively.

$$
\left\{\begin{array}{l}
a_{0}=\frac{2}{T} \int_{-T / 2}^{T / 2} f(x) d x \\
a_{n}=\frac{2}{T} \int_{-T / 2}^{T / 2} f(x) \cos n \omega x d x . \\
b_{n}=\frac{2}{T} \int_{-T / 2}^{T / 2} f(x) \sin n \omega x d x
\end{array}\right.
$$

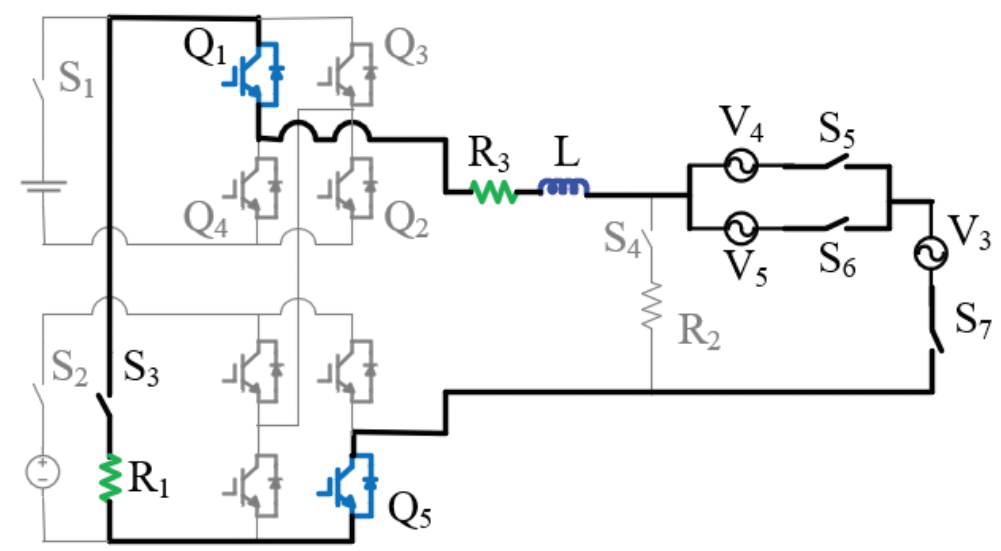

Fig. 5. Schematic of the proposed converter in rectifier mode.

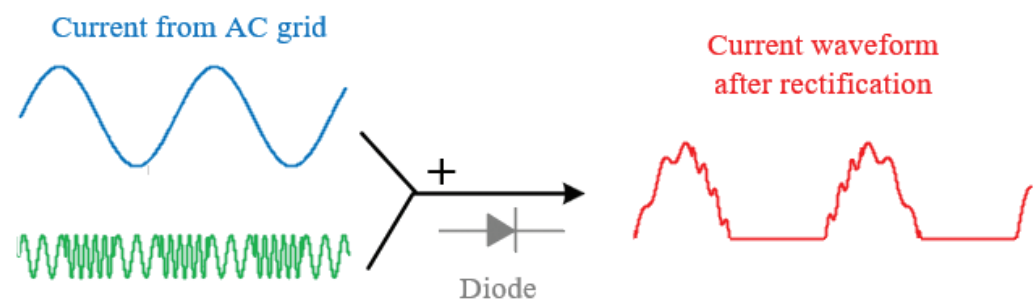

Modulated signal

Fig. 6. The integration process of transmitting signal and power in the rectifier mode. 
Therefore, if a square wave $f(t)$ shown in Eq. (3) is employed as the carrier to modulate the transmission signal in the inverter mode, the Fourier series expansion of $f(t)$ can be expressed as $F(t)$ in Eq. (4).

$$
\begin{aligned}
& f(t)= \begin{cases}0 & -\frac{T}{2} \leq t<0 \\
1 & 0 \leq t \leq \frac{T}{2}\end{cases} \\
& F(t)=\frac{1}{2}+\frac{2}{\pi} \sin \omega t+\frac{2}{3 \pi} \sin 3 \omega t+\frac{2}{5 \pi} \sin 5 \omega t+\frac{2}{7 \pi} \sin 7 \omega t+\cdots+\frac{2}{n \pi} \sin n \omega t
\end{aligned}
$$

Since the first-order harmonic of Eq. (4) has the largest amplitude and has the same frequency as that of the square wave, the extracted first-order harmonic can represent the position of the corresponding carrier in the time domain. Thus, the square carrier used in the inverter mode can be extracted by a bandpass filter. Besides, the sinusoidal carrier employed in the rectification mode can also be extracted by this approach.

An envelope detector is then applied to the demodulated carrier for upper envelope extraction. In this process, the input carrier is first squared and then passed through a low-pass filter. Squaring the carrier can raise half of the signal energy to higher frequencies and transfer the energy of the other half of the signal to the lower frequencies which are close to DC. Next, a low-pass filter is utilised to eliminate the high-frequency energy generated by the squaring process. The remaining curve after filtering is the upper envelope of the carrier. After comparing the upper envelope with a threshold value, the transmitted data can finally be restored by down-sampling the curve after the comparison process at the initial data rate. Figure 8 shows a schematic diagram of the demodulation process of

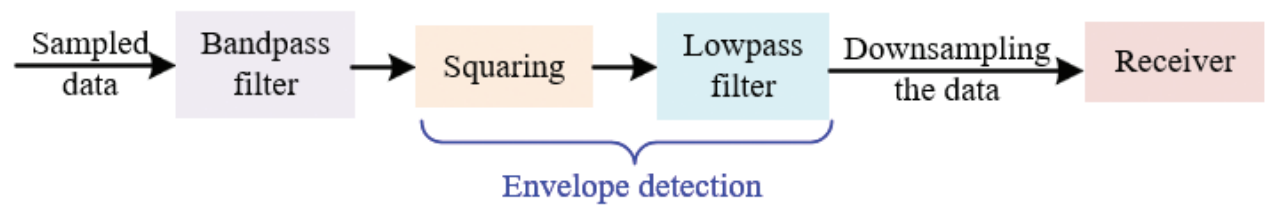

Fig. 7. The signal demodulation process of the proposed model.

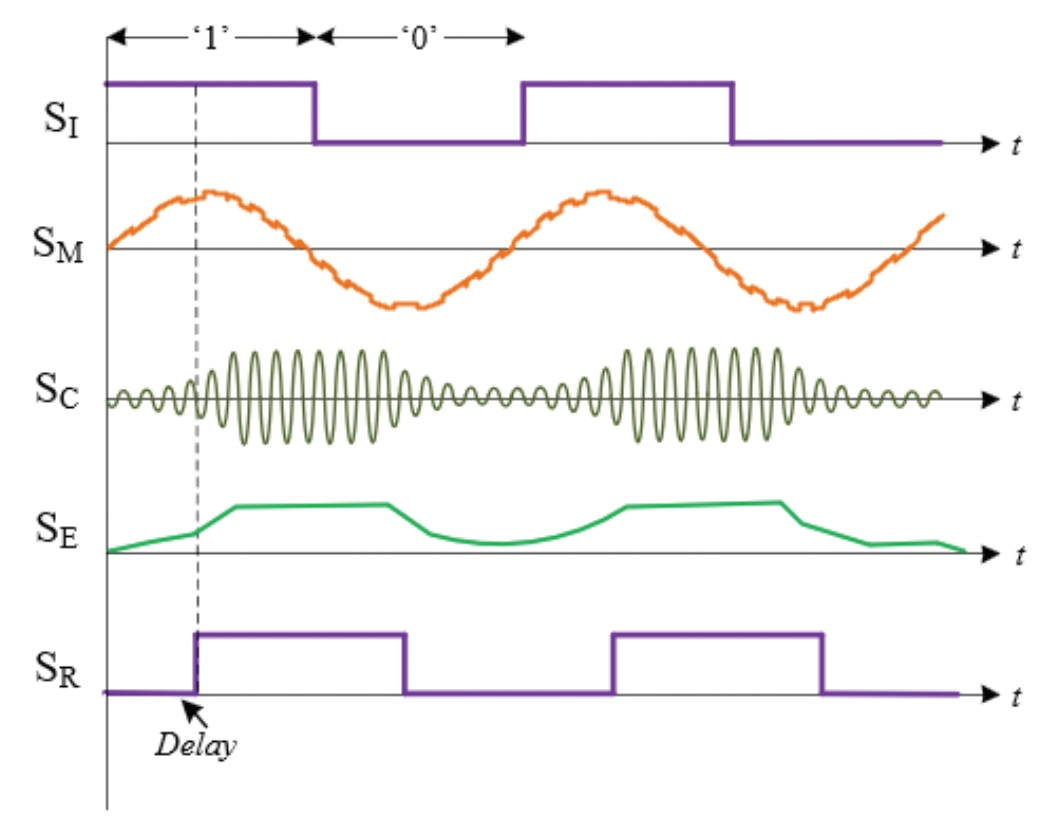

Fig. 8. The schematic diagram of the demodulation process of the signal '1010', where $S_{1}$ is the initial transmitted signal, $S_{M}$ is the integrated current and signal waveform, $S_{C}$ represents the demodulated carrier for digital '1', $S_{E}$ exhibits the upper envelope of the demodulated carrier and $S_{R}$ is the restored signal. 
the signal '1010', where $S_{1}, S_{M}, S_{C}, S_{E}$ and $S_{R}$ represent the original signal, integrated current and signal waveform, demodulated carrier, upper envelope and restored signal, respectively.

\section{Simulation Results and Analysis}

\subsection{Parameters and Output Waveforms}

A simulation model of the proposed converter topology is implemented in MATLAB/Simulink using the power and signal transmission method demonstrated in Section 3. The parameters utilised in this model are given in Table 1. The DC and the AC sides use a $200 \mathrm{~V}$ DC voltage source and a $200 \mathrm{~V}$ AC voltage source, respectively, as the power supply for the power conversion part. The signal carriers of the DC and the AC sides are generated by an additional $10 \mathrm{~V}$ DC voltage source and two additional $10 \mathrm{~V}$ AC voltage sources, respectively. Since the amplitude of the carrier generated by the $10 \mathrm{~V}$ power supply will not greatly distort the current waveform of the power conversion section and since the carrier energy is large enough to be detected in the demodulation process, it is appropriate to employ a $10 \mathrm{~V}$ voltage source for carrier generation. In this model, the inherent impedance and inductance of the transmission line are set to $2 \Omega$ and $200 \mu \mathrm{H}$, respectively. When the topology works in inverter mode and only the 'data' converter is considered, its rated power can be calculated by the following equation:

$$
P_{i d}=U_{i d} \times I_{i d} \times D=10 \times 5 \times 50 \%=25 \mathrm{~W}
$$

where $D$ is the duty ratio $(50 \%)$ of the converter switches. Besides, the instantaneous power of the power converter can be obtained with the following formula:

$$
\begin{aligned}
P_{i p}=U_{i p} \times I_{i p} & =U_{m} \sin \omega t \times I_{m} \sin \omega t \\
& =20000(1-\cos 200 \pi t)
\end{aligned}
$$

Since the inductance value of the transmission line in the simulation model is much smaller than the impedance value, the influence of the inductance on the power is neglected in Eq. (6) to simplify the analysis. Therefore, the rated power of the power converter is approximately equal to $10 \mathrm{~kW}$. Similarly, when the topology operates in rectifier mode, the rated power of the 'data' converter is denoted by Eq. (7). Additionally, the expression of the instantaneous power of the power converter is the same as that presented in Eq. (6).

$$
P_{r d}=U_{r d} \times I_{r d}=\frac{1}{2} U_{m} I_{m}=25 \mathrm{~W}
$$

It can be observed that the rated power of the 'data' converter is smaller than that of the power converter. However, since the 'data' converter is connected in series with the power converter, it actually conducts full current and its actual power rating is greater than the derived value. Specifically, if the current flowing in the power converter is taken into account, for both inverter mode and rectifier mode, using Eqs (5) and (7) we can approximate the rated power of the 'data' converter as $525 \mathrm{~W}$.

Table 1. Parameters value utilised in the simulation model.

\begin{tabular}{ll}
\hline Parameter name & Value \\
\hline \hline AC power source frequency & $50 \mathrm{~Hz}$ \\
DC voltage source for power conversion & $200 \mathrm{~V}$ \\
AC voltage source for power conversion & $200 \mathrm{~V}$ \\
DC side carrier voltage & $10 \mathrm{~V}$ \\
AC side carrier voltage & $10 \mathrm{~V}$ \\
DC side carrier frequency for signal modulation & $4 \mathrm{kHz}$ for ' 1 ' and $5 \mathrm{kHz}$ for ' 0 ' \\
DC side carrier frequency for AC current modulation & $20 \mathrm{kHz}$ \\
Modulated AC current frequency & $50 \mathrm{~Hz}$ \\
AC side carrier frequency & $1.5 \mathrm{kHz}$ for ' 1 ' and $3 \mathrm{kHz}$ for ' 0 ' \\
\hline
\end{tabular}


After establishing the simulation model and setting the parameters, the output current waveform of the AC grid is obtained as shown in Figure 9. The simulation lasts for $2 \mathrm{~s}$, in which the converter works in the inverter mode for the former $1 \mathrm{~s}$ and in the rectifier mode for the latter $1 \mathrm{~s}$. The modulated signals are superimposed on the current waveform as expected, which proves that the proposed energy and signal integrated transmission approach is feasible.

\subsection{Signal Transmission}

An 8-bit signal ' 11010001 ' with the data rate of 16 bps is transmitted while the converter operates in the inverter mode. Before modulating the signal, a 4-bit cyclic redundancy code (CRC) is added at the end of each 8-bit data string for error checking. Specifically, the 4-bit CRC code created with the polynomial $x^{4}+x^{3}+x^{2}+x+1$ is ' 0100 '. Then the 12-bit data frame '110100010100' is transmitted and divided by the divisor '11111' at the receiver by modulo-2 division. If there is no remainder after the division operation, it indicates that the transmitted frame is correct. Since the added CRC code does not take extra time to transmit, the actual data rate in the first $1 \mathrm{~s}$ of the simulation is 24 bps. The original 8-bit signal and the 12-bit combined data frame are exhibited in the curves $S_{\circ}$ and $S_{p}$ in Figure 10, respectively.

The $4 \mathrm{kHz}$ and $5 \mathrm{kHz}$ carriers are employed to modulate digital ' 1 ' and digital ' 0 ', respectively. After the modulated signal is transmitted along with the energy, the $4 \mathrm{kHz}$ carrier is extracted from the current waveform at the receiving terminal using a bandpass filter. The extracted $4 \mathrm{kHz}$ carrier is presented in the curve $\mathrm{S}_{T}$ of Figure 10 . Next, the upper envelope of the demodulated $4 \mathrm{kHz}$ carrier is obtained by the envelope detection process, and the result is shown in the curve $S_{Y}$ in Figure 10. Since the attenuation at the cut-off frequencies of the bandpass filter is set at $6 \mathrm{~dB}$, the attenuation ratio $x$ is obtained as 0.5 based on Eq. 8 .

$$
20 \log x=-6
$$

Therefore, the amplitude of the extracted carrier curve $S_{T}$ in the passband frequencies region is twice of its amplitude in the cut-off frequencies region. Moreover, since the amplitude of the carrier envelope obtained by envelope detection has not changed significantly, the average value '1.5' of the amplitudes of two different frequency regions is taken as the threshold with which to convert the carrier envelope into a digital signal. If the carrier envelope is higher than the threshold value, the digital signal ' 1 ' will be generated. Otherwise, the digital signal ' 0 ' will be generated. The digital signal after comparison is displayed as $S_{Q}$ in Figure 10. Finally, the curve $S_{Q}$ is sampled using the original data rate for signal recovery, and the recovered signals with and without CRC code are represented by curves $S_{F}$ and $S_{G}$ in Figure 10, respectively. After comparing curve $S_{F}$ with the original data string $S_{P}$, a delay that is mainly caused by the filtering process can be found. Thus, the recovered data actually begins at the second sampling point (simulation time $t=1 / 12 \mathrm{~s}$ ) of curve $\mathrm{S}_{\mathrm{F}}$. Moreover, since the signal is transmitted in frames, the restored signal is manually delayed for one frame to ensure the consistency of the data size of each frame. When the CRC code is removed, the restored signal has the same data rate as the original signal $\mathrm{S}_{\mathrm{o}}$, and it appears after $t=1 \mathrm{~s}$, as shown in curve $\mathrm{S}_{\mathrm{G}}$ in Figure 10 .

Similarly, the 16-bps signal ' 00101110 ' is transmitted through the converter in the rectifier mode using the same modulation and demodulation methods as those applied in the inverter mode. The original 16-bps signal and the signal combined with 4-bit CRC code are shown in curves $S_{J}$ and $S_{K}$ in Figure 11, respectively. The generated CRC

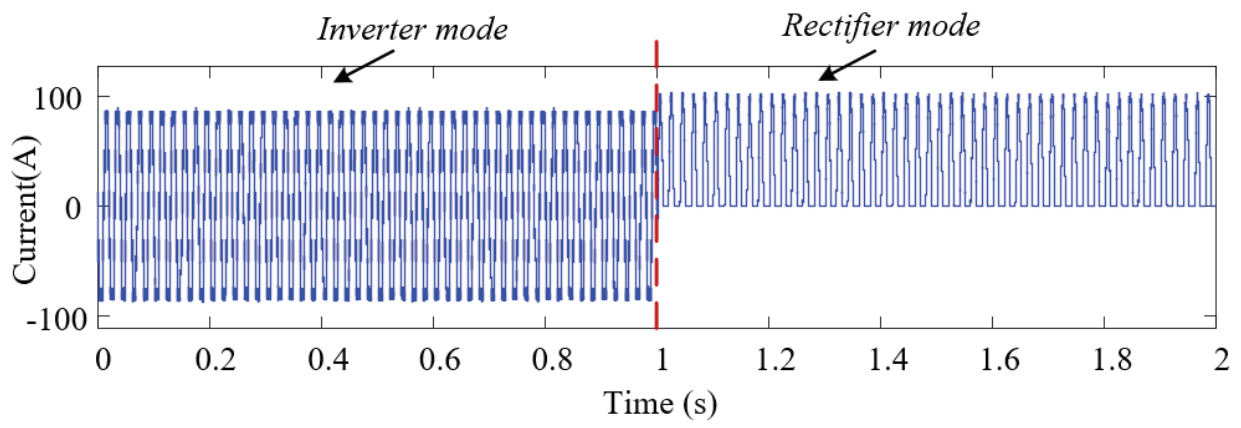

Fig. 9. The output current waveform of the AC grid. 

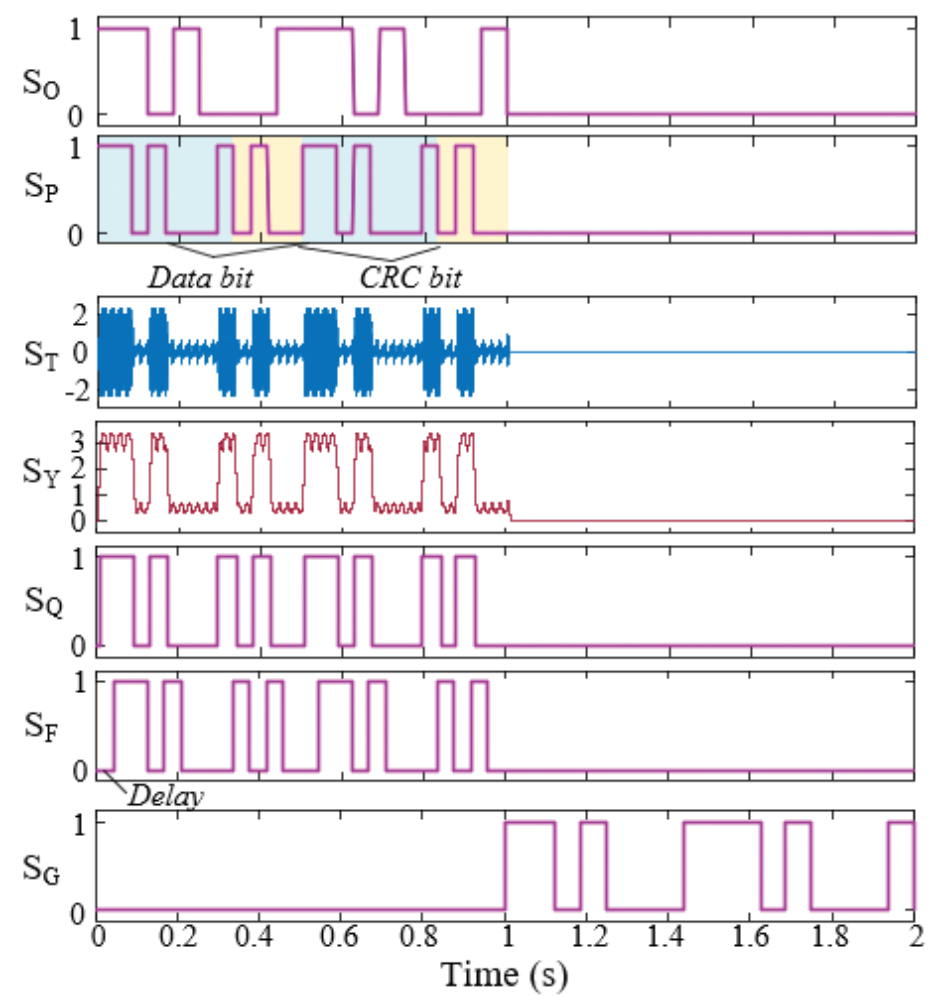

Fig. 10. The signal transmitted in inverter mode, where $S_{O}$ is the original signal, $S_{P}$ is the signal combined with $C R C$ code, $S_{T}$ is the demodulated carrier for digital ' 1 ', $S_{Y}$ is the upper envelope of the extracted carrier, $S_{Q}$ is the curve after comparison, $S_{F}$ is the resampled signal and $S_{G}$ is the received signal without CRC code. CRC: cyclic redundancy code.

code for the 8-bit signal using the polynomial $x^{4}+x^{3}+x^{2}+x+1$ is '1000'. Since the converter in the simulation works in the rectifier mode between 1 and $2 \mathrm{~s}$, considering the signal delay characteristics, Figure 11 shows the simulation results between 1 and $3 \mathrm{~s}$. After the modulated signal is transmitted along with the current, the demodulated carrier for digital ' 1 ' and its upper envelope are obtained as displayed in curves $S_{L}$ and $S_{x}$ in Figure 11, respectively. Finally, the recovered signal $S_{W}$ is acquired by resampling curve $S_{U}$, and the received signal with 4-bit CRC code removed is exhibited in curve $S_{\mathrm{N}}$ in Figure 11.

\subsection{Data Transmission Capability}

To determine the maximum signal rate that the proposed method can transmit, the data transmission rate in the two modes is gradually increased to investigate the relationship between the bit error rate (BER) and the signal rate. After calculating the BER by comparing the initially transmitted and recovered data strings bit by bit, the BER curve is sketched as shown in Figure 12. It can be observed from Figure 12 that the BER starts rising when the data rate is higher than $120 \mathrm{bit} / \mathrm{s}$ in the inverter mode. Besides, the error bits in the rectifier mode begin to appear when the data rate is higher than $48 \mathrm{bit} / \mathrm{s}$. Since the modulated signal is superimposed on the current, and the designed converter uses diodes to achieve half-wave rectification, the signal cannot propagate when the diodes are reverse biased, and this results in a high BER in the rectifier mode. Specifically, a $50 \mathrm{~Hz} \mathrm{AC}$ power supply is used for rectification in this simulation model; thus, the current period is $0.02 \mathrm{~s}$ and the theoretical maximum signal transmission rate is $100 \mathrm{bit} / \mathrm{s}$. Considering the delay of the filter and the error of the envelope detector, the appropriate actual data rate is much smaller than the theoretical value. Therefore, the accuracy of the restored signal can be improved by employing filters with higher orders. Although the proposed signal transmission method is slightly insufficient in terms of the data rate when compared with the conventional PLC approach, the simplified circuit structure can satisfy the requirements of power and signal bidirectional transmission while reducing the cost. Thus, it is beneficial to apply this method to AC microgrids. 

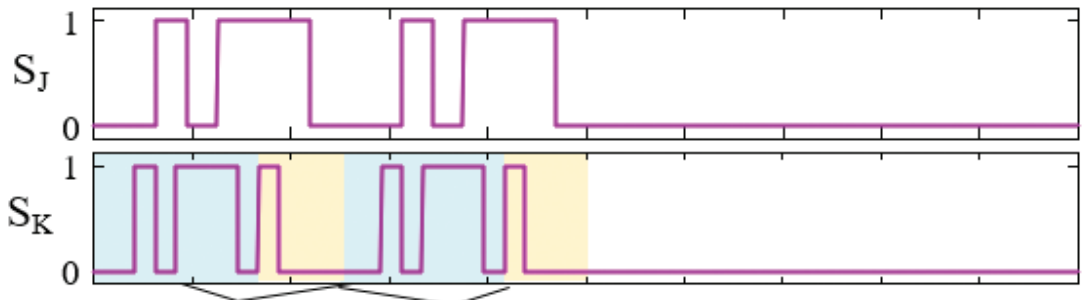

Data bit CRC bit
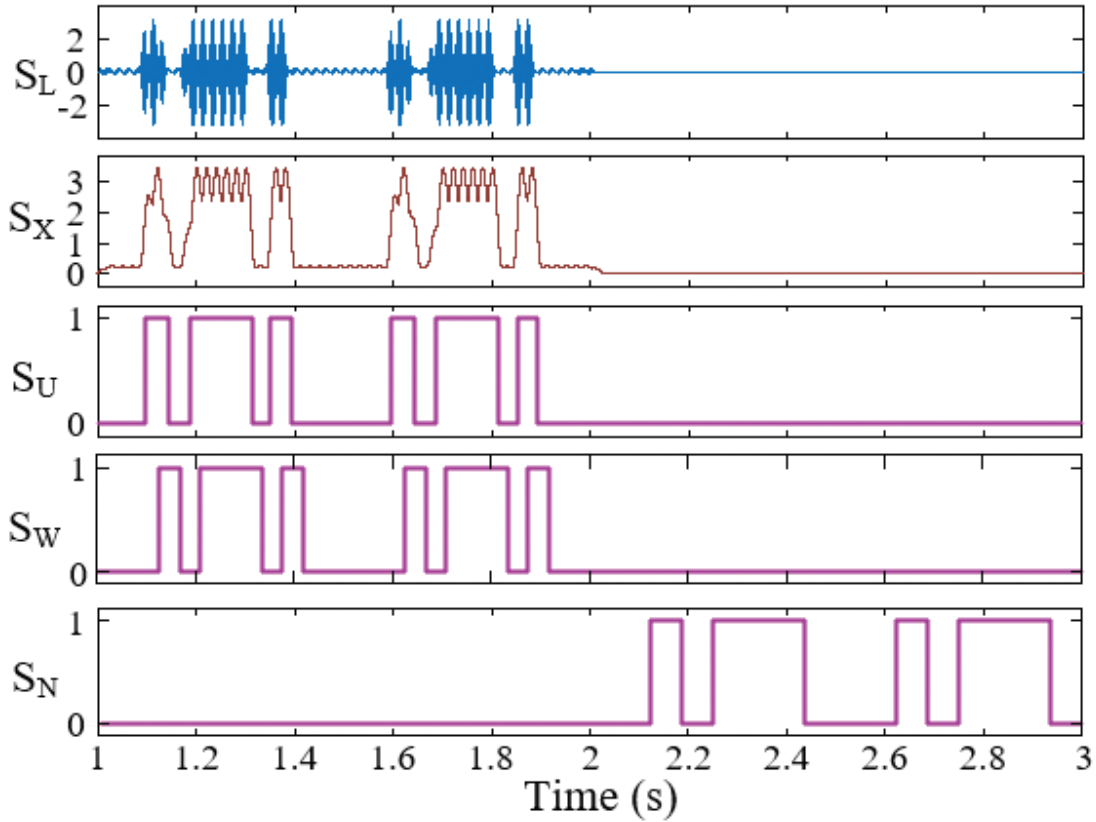

Fig. 11. The signal transmitted in rectifier mode, where $S_{\text {, is }}$ is the original signal, $S_{k}$ is the signal combined with $C R C$ code, $S_{b}$ is the demodulated carrier for the digital signal ' 1 ', $\mathrm{S}_{\mathrm{X}}$ is the upper envelope of the extracted carrier, $\mathrm{S}_{\mathrm{U}}$ is the curve after comparison, $\mathrm{S}_{\mathrm{W}}$ is the resampled signal and $\mathrm{S}_{\mathrm{N}}$ is the received signal after $\mathrm{CRC}$ code removed. $\mathrm{CRC}$ : cyclic redundancy code.

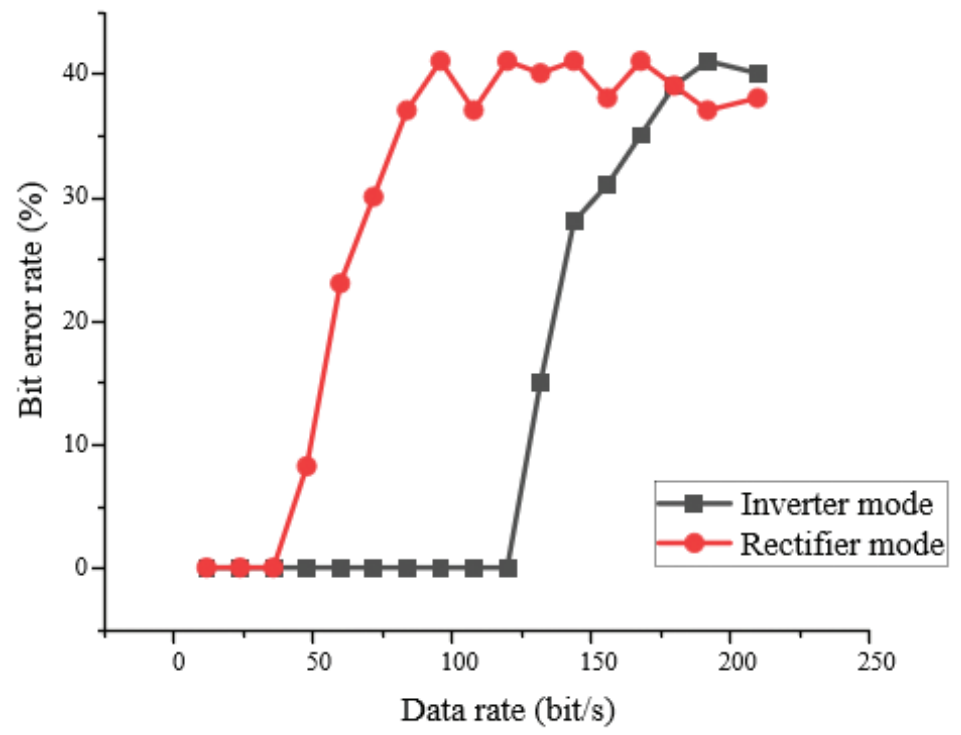

Fig. 12. The relationship between the signal bit rate and error rate when the converter operates in two different modes. 


\section{Conclusion}

A bidirectional DC-AC converter topology is proposed in this paper to realise two-way communication in microgrids. In this topology, the communication signal is modulated by the power switches using the FSK method, by which the communication signal can be superimposed on current and transmitted through the AC grid. Two series-connected $\mathrm{H}$-bridge converters are employed to convert the current type from DC to AC. Conversely, the AC current is rectified through the anti-parallel diodes of the IGBT switches of the H-bridge converter. Since the proposed method uses power switches for signal modulation, the signal couplers used in the conventional PLC-based microgrid are omitted. Therefore, such a method has the characteristics of simple circuit structure and low cost. After implementing the proposed converter model in MATLAB/Simulink, the feasibility of modulating the communication signal through power switches is verified. Moreover, when the power frequency is $50 \mathrm{~Hz}, 120 \mathrm{bit} / \mathrm{s}$ and $48 \mathrm{bit} / \mathrm{s}$, signals can be transmitted in the inverter mode and rectifier mode, respectively.

\section{References}

Chakraborty, S., Weiss, M. D. and Simoes, M. G. (2007). Distributed Intelligent Energy Management System for A Single-Phase High-Frequency AC Microgrid. IEEE Transactions on Industrial Electronics, 54(1), pp. 97-109.

Choi, H. J. and Jung, J. H. (2017). Enhanced Power Line Communication Strategy for DC Microgrids Using Switching Frequency Modulation of Power Converters. IEEE Transactions on Power Electronics, 32(6), pp. 4140-4144.

Costa, L. G. D. S., Picorone, A. A. M., de Queiroz, A. C. M., Costa, V. L. R. and Ribeiro, M. V. (2015). Projeto e caracterização de acopladores para power line communications. In: Proceedings of XXXIII Simpósio Brasileiro de Telecomunicações. Brazil, 2015.

Costa, L. G., de Queiroz, A. C. M., Adebisi, B., da Costa, V. L. R. and Ribeiro, M. V. (2017). Coupling for Power Line Communications: A Survey. Journal of Communication and Information Systems, 32(1).

Fang, X., Misra, S., Xue, G. and Yang, D. (2011). Smart Grid - The New and Improved Power Grid: A Survey. IEEE Communications Surveys \& Tutorials, 14(4), pp. 944-980.

Ghorbanian, M., Dolatabadi, S. H., Masjedi, M. and Siano, P. (2019). Communication in Smart Grids: A Comprehensive Review on the Existing and Future Communication and Information Infrastructures. IEEE Systems Journal, 13(4), pp. 4001-4014.

Gungor, V. C., Sahin, D., Kocak, T., Ergut, S., Buccella, C., Cecati, C. and Hancke, G. P. (2011). Smart Grid Technologies: Communication Technologies and Standards. IEEE Transactions on Industrial Informatics, 7(4), pp. 529-539.

Hau, L. C., Lee, J. V., Chuah, Y. D. and Lai, A. C. (2013). Smart Grid-the Present and Future of Smart

Physical Protection: A Review. International Journal of Energy, Information and Communications, 4(4), pp. 43-54.

Kabalci, Y. (2016). A Survey on Smart Metering and Smart Grid Communication. Renewable and Sustainable Energy Reviews, 57, pp. 302-318.

Lee, J. J., In, D. S., Oh, H. M., Shon, S. and Nam, D. H. (2010). Neutral Inductive Coupling for Improved Underground Medium Voltage BPLC. In: ISPLC2010. IEEE. March 2010.

Lo, C. H. and Ansari, N. (2011). The Progressive Smart Grid System from Both Power and Communications Aspects. IEEE Communications Surveys \& Tutorials, 14(3), pp. 799-821.

Lu, Z., Lu, X., Wang, W. and Wang, C. (2010). Review and Evaluation of Security Threats on the Communication Networks in the Smart Grid. In: 2010-Milcom 2010 Military Communications Conference. IEEE. October 2010.

Mocanu, E., Nguyen, P. H., Kling, W. L. and Gibescu, M. (2016). Unsupervised Energy Prediction in a Smart Grid Context Using Reinforcement Cross-Building Transfer Learning. Energy and Buildings, 116, pp. 646-655.

Stefanutti, W., Mattavelli, P., Saggini, S. and Panseri, L. (2006). Communication on Power Lines Using Frequency and Duty-Cycle Modulation in Digitally Controlled DC-DC Converters. In: IECON 200632nd Annual Conference on IEEE Industrial Electronics. IEEE. November 2006.

Stefanutti, W., Saggini, S., Mattavelli, P. and Ghioni, M. (2008). Power Line Communication in Digitally Controlled DC-DC Converters Using Switching Frequency Modulation. IEEE Transactions on Industrial Electronics, 55(4), pp. 1509-1518. 
Sun, Q., Ge, X., Liu, L., Xu, X., Zhang, Y., Niu, R. and Zeng, Y. (2011). Review of Smart Grid Comprehensive Assessment Systems. Energy Procedia, 12, pp. 219-229.

Swana, Z. W., van Rensburg, P. A. J. and Ferreira, H. C. (2015). Is Resistive Coupling Feasible for the Reception of Power-Line Communications Data? In: 2015 IEEE International Symposium on Power Line Communications and Its Applications (ISPLC). IEEE. March 2015.

Tripathi, K., Shrivastava, S. and Banarjee, S. (2020). Review in Recent Trends on Energy
Delivery System and Its Issues in Smart Grid System. In: Computing Algorithms with Applications in Engineering. Springer, pp. 117-125.

Wang, R., Lin, Z., Du, J., Wu, J. and He, X. (2016). Direct Sequence Spread Spectrum-Based PWM Strategy for Harmonic Reduction and Communication. IEEE Transactions on Power Electronics, 32(6), pp. 4455-4465.

Wang, W., Xu, Y. and Khanna, M. (2011). A Survey on the Communication Architectures in Smart Grid. Computer Networks, 55(15), pp. 3604-3629. 